\title{
Eisenstein contemporain : entretien avec Antonio Somaini
}

\section{Bruno Bueno Pinto Leites}

Doutorando; Universidade Federal do Rio Grande do Sul

bleites2003@hotmail.com

\section{Résumé}

Entretien avec Antonio Somaini à propos des films, des écrits et des dessins de Sergei Eisenstein. Somaini est actuellement un des plus grands spécialistes de l'œuvre d'Eisenstein. À l'entretien, nous parlons de nombreux sujets: les défis de traduire les ouvrages d'Eisenstein, les phases de sa carrière, le développement de sa pensée, la relation de ses films avec ses écrits, sa pratique du dessin et sa passion pour les dessins de Walt Disney. C'est un long entretien dans laquelle nous parlons aussi des principaux concepts d'Eisenstein, tels que l'extase, le pathos, l'organicité, l'attraction, la régression et la plasmaticité. Somaini nous parle également de la spécificité de son travail et de l'urgence de reprendre Eisenstein. D'un côté, il faut le faire sortir de l'histoire du cinéma exclusivement et l'inclure dans le réseau le plus vaste des arts et de la pensée des années 1920, 1930 et 1940. De l'autre côté, il faut penser son projet comme essentiellement contemporain. Comme Somaini l'a dit, ce que nous pensons beaucoup aujourd'hui, le dispositif cinématographique, le rapport entre le cinéma et l'histoire de l'art, l'influence des images sur l'ensemble de l'expérience humaine, cela a toujours été le problème d'Eisenstein.

\section{Mots-clés}

Eisenstein. Somaini. Extase. Pathos. Plasmaticité.

\section{Présentation de l'entretien}

Le professeur Somaini m'a reçu dans un petit bureau à l'Université Nouvelle Sorbonne - Paris 3, au printemps 2015. À cette époque-là, je n'étais qu'un des nombreux étudiants étrangers qui suivaient ses cours à l'Université. On a accordé de faire un entretien autour du sujet « les films et les écrits de Sergei Eisenstein ». L'oeuvre d’Eisenstein, comme nous l'apprend Somaini dans cet entretien, nous est toujours nécessaire, car elle met en 
relief des questions qui sont encore très contemporaines dans la théorie des médias, des images et du cinéma.

Somaini, professeur italien qui enseigne à Paris, est spécialiste de l'œuvre de Sergei Eisenstein. Son livre « Ejzenštejn. Il cinema, le arti, il montaggio », publié originalement en 2011 en italien, doit être lancé en 2016 en français et en anglais par d'importantes maisons d'éditions : la CNRS Éditions en France et The University of Illinois Press aux États-Unis.

Somaini a établi, avec Naum Kleiman, le livre « Notes pour une histoire générale du cinéma » (EISENSTEIN, 2014a), qui est également paru en 2014 au Brésil (EISENSTEIN, 2014b). Dans ce livre-là, il y a un essai approfondi de Somaini sur Eisenstein, sous le titre de «Généalogie, morphologie, anthropologie des images, archéologie des médias » (SOMAINI, 2014).

À propos de l'œuvre de Somaini sur Eisenstein, premièrement il faut bien souligner qu'elle contient renferment une foule de renseignements en termes de contenu informatif, historique et bibliographique. Cela est remarquable dans son chef-d'oeuvre à ce sujet, « Ejzenštejn. Il cinema, le arti, il montaggio » (SOMAINI, 2011), et aussi dans «Généalogie, morphologie, anthopologie des images, archéologie des médias » (SOMAINI, 2014). Ensuite, il faut souligner les analyses et l'approche de Somaini : il met Eisenstein en rapport avec son époque et avec la nôtre. Comme dit Somaini dans cet entretien, Eisenstein n'a jamais rencontré Walter Benjamin, Aby Warburg et Ernest Bloch, mais sa pensée est en profonde relation avec la leurs et avec celle d'autres artistes et intellectuels des années 1920, 1930 et 1940.

En ce qui concerne notre époque, Somaini nous enseigne qu'Eisenstein est contemporain :

« Et alors là il me semble qu'Eisenstein a une actualité remarquable, parce que, par exemple, dans une phase comme la nôtre, dans laquelle nous nous interrogeons beaucoup sur les transformations du dispositif cinématographique, sur le rapport entre le cinéma et l'histoire des arts, des arts plastiques etc., toute la pensée d'Eisenstein, si on veut, a tourné aussi autour de cette question. »

Selon Somaini, Eisenstein est contemporain parce qu'il fait une pratique des images qu'on pourrait qualifier comme anthropologique, c'est-à-dire, une pratique des images en leurs relations avec nos besoins, nos impulsions, nos nécessités, enfin, de l'image et ses effets sur l'ensemble de l'expérience humaine. 
Ainsi, il est plusieurs fois contemporain. C'est comme cela, à partir de Somaini, qu'on peut mieux connaître Eisenstein.

Dans votre livre sur Eisenstein, vous dites que dans chacune des dernières œuvres d'Eisenst ein il y a un sujet principal. Puis, dans "Metod " cela serait la pensée prélogique, dans la " Nonindifférente nature " cela serait l'extase, etc. Je voudrais savoir comment vous comprenez le concept d'attraction dans l'ensemble de l'œuvre d'Eisenstein, et si vous êtes d'accord qu'il y aurait deux grandes phases chez lui : une première liée aux attractions et une deuxième liée au pathos, à l'extase et à la pensée prélogique.

Alors, je pense qu'Eisenstein, au-delà de la grande diversité qu'on trouve dans ses écrits, de la grande diversité de références théoriques, historiques etc., s'est toujours occupé essentiellement d'une seule et même question. Ça veut dire : comment produire des œuvres d'art, soit à travers le cinéma, soit à travers le théâtre ou d'autres formes de représentation... des œuvres d'art capables d'agir sur le corps et l'esprit du spectateur. Sa préoccupation principale a été toujours celle de l'efficacité de l'œuvre d'art.

Pour penser cette efficacité il a élaboré dans ses écrits plusieurs concepts et plusieurs théories. Le premier concept qu'on y trouve, c'est celui du mouvement expressif. Donc, comment faire ainsi que l'acteur de théâtre, et en 1922 Eisenstein était encore actif dans le milieu du théâtre, comment faire ainsi que le mouvement, le geste de l'acteur de théâtre soit efficace, donc capable de transmettre des émotions, des impulsions au public. De cette théorie du mouvement expressif naît la théorie des attractions, qu'il élabore entre 1923 et 1924, quand il est en train de passer du théâtre au cinéma. Et l'idée des attractions est que le spectacle théâtrale ou cinématographique doit contenir des moments agressifs, des moments violents, des moments capables d'exercer quelque impression, au sens presque littéral et physique du terme, sur le corps et l'esprit du spectateur. Il s'agit dans ce moment-là d'orienter le spectateur du théâtre ou du cinéma dans une direction idéologique voulu. Et l'attraction est l'instrument de cette orientation.

Après, Eisenstein dans ses écrits successifs, élaborera d'autres concepts et d'autres théories, mais toujours pour aborder la même question : comment produire des spectacles efficaces. Et donc de l'attraction on passera à l'idée de montage intellectuel, ou encore à l'idée de montage conflictuel, ça veut dire, la capacité de produire des formes qui contiennent quelque sorte de conflit, de dynamique, de friction à leur intérieur et qui soit capable, à travers ses conflits, d'activer l'esprit et le corps du spectateur. 
Après le voyage au Mexique, le séjour à Paris entre 1929 et 1930 et le retour à Moscow dans les premières années 1930, Eisenstein commencera à s'intéresser d'autant plus à l'anthropologie, à l'ethnologie. Donc, à tous les écrits sur la mentalité primitive, la pensée prélogique, comme ceux de James Frazer, «Le Rameau d’or », ou encore Lévy-Bruhl, sur la mentalité primitive ou ce que Lévy-Bruhl appelait « Les fonctions mentales dans les sociétés inférieures », le titre de son livre de 1910. Dans ses écrits après le Mexique, la même question qui l'a toujours occupée, comment agir sur le spectateur, est traitée à travers d'autres outils théoriques, d'autres concepts, qui dérivent de ses lectures.

Notamment, le concept qui à mon avis est central dans tous les textes qui feront partie du projet de livre appelé « Metod», le concept central sera le concept de régression. Comment produire des formes qui dérivent leurs efficacités de la présence à leurs intérieurs de signes, de traces, disons, d'une mentalité prélogique ou de forme de vie biologique, primordiale, originaire. Eisenstein s'intéresse à la régression selon des perspectives très variées : régression psychique vers des états mentaux initiaux, régression biologique vers des formes de vie primitives, primordiales. Et il cherche dans ce vaste fond du primitive, du prélogique, du primordial, il cherche des formes qui puissent être réactivées dans le milieu artistique pour donner plus d'efficacité à la forme artistique elle-même. Donc il s'agit des nouveaux concepts, des nouvelles perspectives, mais toujours pour penser la même question. Et ceci concerne, comme je disais, le livre « Metod ».

Si après on prend en considération d'autres livres, qui sont tous restés inachevés, comme " Montage », autour de 1937/1938, ou encore "La non-indifférente nature », dans les années 1940, on trouve que d'autres concepts sont utilisés toujours pour penser cette même question. Dans le livre « Montage », c'est le concept d'Obraz. L'idée est que le montage doit produire des images, qu'il appelle Obraz, qui soient à même temps douées d'un sens général, capable de synthétiser à leur intérieur un concept ou une idée générale, complexe, mais à même temps riche du point de vue émotionnel, émotif. Il s'agit donc de produire des images globales, synthétiques, encore une fois capables d'influencer le corps et l'esprit du spectateur.

Finalement, dans « La non-indifférente nature » les concepts qui seront au centre de la pensée d'Eisenstein seront les concepts d'extase et de pathos. Et là encore il s'agit de théoriser ces concepts et de les utiliser, disons, comme des principes de référence pour produire des formes efficaces. Donc, à mon avis la pensée d'Eisenstein est caractérisée d'un côté par une grande diversité de références, une évolution, le passage d’un concept à l'autre, 
d'une référence à l'autre, mais en même temps par un fil rouge qui la traverse complètement et qui est cette question de l'efficacité de la forme, de l'efficacité du montage. Le montage, comme je dis dans un de mes textes ${ }^{1}$, est conçu par Eisenstein comme un instrument pour monter le spectateur, pour arriver à concevoir le spectateur lui-même comme objet de montage, comme quelque chose qui doit être transformée par l'œuvre d'art.

Toutes les dernières œuvres d'Eisenstein sont restées inachevées ou, au moins, la majorité. En tout cas, je vous pose la question sur le processus de traduction de ses dernières œuvres: Eisenstein était déjà un réalisateur très connu aux années 1930 et 1940, il était déjà traduit en anglais aux années 1940, je crois, mais il y a encore aujourd'hui le défi de connaître l'œuvre théorique d'Eisenstein, de la traduire, etc. Est-ce que c'est seulement à cause de la barrière linguistique ou peut-être à cause du style fragmentaire d'Eisenstein ou y aurait-il une autre cause, liée au sujet même de sa pensée ?

Donc, pendant sa vie, Eisenstein a publié plusieurs textes dans des revues ou des livres, etc., mais il n'est jamais arrivé à achever complètement un des plusieurs projets de livres sur lesquels il avait travaillé. $\mathrm{Ni}$ «Montage», ni «La non-indifférente nature», ni « Metod», ni « Notes pour une histoire générales du cinéma » ont été achevés. Donc, ceux qu'il avait publié pendant sa vie étaient des textes séparés, mais qui faisaient partie de plus vastes projets, qui n'ont été jamais achevés.

Ceux qu'on a connu à l'étranger ont été les deux livres publiés en anglais avec les titres «Film form» et «Film sens $»^{2}$. Et dans les deux cas, il s'agit de livres qui mettent ensemble plusieurs textes, mais encore une fois qui ne réfléchissent pas un projet de livre complet conçu par Eisenstein. Il avait publié pendant sa vie des textes en français aussi. Par exemple, il a publié deux ou trois articles en traduction française pendant qu'il était à Paris entre 1929 et 1930³. Mais aucun de ces livres n'a été achevé.

Or la difficulté que nous avons avec les traductions de ses textes est due à plusieurs causes. D'un côté, c'est le fait que le travail qu'a été fait en Russie sur les textes d'Eisenstein a abouti à plusieurs éditions. Dans les années 1960, on a fait une édition en six volumes, qui a été après dépassée par d'autres volumes parus entre la fin des années 1980 et les premières dix années de 2000. Ce sont de gros volumes édités par Naum Kleiman qui ont

\footnotetext{
1 Il s'agit de "Ejzenštejn. Il cinema, le arti, il montaggio". Voir surtout le deuxième chapitre, "Modellare lo spettatore': il montaggio delle attrazioni” (SOMAINI, 2011, p.17-37)

2 “The film sense" (EISENSTEIN, 1943). "Film form: Essays in film theory” (EISENSTEIN, 1949).

3 «Les gendarmes à la Sorbonne » (EISENSTEIN, 1930a). « Les principes du nouveau cinéma russe » (EISENSTEIN, 1930b).
} 
finalement mis à jour des œuvres comme « Metod», comme « Montage » et comme «La nonindifférente nature », dans une version plus large de celle qu'on connaissait.

\section{C'était déjà à la fin des années 1980 ?}

Ces trois grands volumes, dont «Montage», qui est un seul volume, "La nonindifférent nature », en deux volumes, et « Metod», en deux volumes, sont parus entre, voilà, disons, entre la fin des années 1990 et 2005, 2006, à peu près ${ }^{4}$.

Donc, en Russie il y a eu ce travail d'édition qui a fait paraître différentes éditions des mêmes textes, parce que « La non-indifférente nature » a été publiée une première fois dans l'édition en six volumes, dans les années 1960, et après, une deuxième fois, en 2006, dans cette nouvelle édition. Donc, déjà en Russie il y a un problème. Il y a des éditions qui sont parues plusieurs fois et des écrits qui ont été, disons, présentés au public petit à petit. Et ce travail qui s'est étalé pendant des dizaines d'années en Russie a fait ainsi que les éditions étrangères soient traduites parfois des textes qui après ont été républiés de différentes formes en Russie.

Par exemple, en France, François Albera, qui est un des plus grands chercheurs sur l'œuvre d'Eisenstein a publié entre la fin des années 1970 et le début des années 1980, deux volumes, qui s'appellent « Cinématisme » (EISENSTEIN, 1980/2009) et « Le mouvement de l'art» (EISENSTEIN, 1986) qui sont des recueils des textes différents d'Eisenstein. Ils ont été d'une grande importance pour la réception d'Eisenstein en France, mais qui ne réfléchissent pas les livres conçus par Eisenstein. Ils sont un peu comme des anthologies de textes. À mon avis, l'édition la plus complète et la mieux organisée qu'on a en ce moment est l'édition italienne, parce qu'il s'agit d'une édition en plusieurs volumes...

\section{Est-ce qu'il y a déjà « Metod » en italien ?}

Il paraîtra bientôt. La traduction est déjà complétée, on attend seulement que l'éditeur le fasse paraître. Donc, fin 2016 ou au plus tard 2017 on aura « Metod » en Italie. Ce sera la première traduction.

Et voilà, l'édition italienne, en plusieurs volumes, a déjà «La non-indifférente nature », les textes sur la couleur, « Montage», plusieurs textes sur le montage des années

\footnotetext{
4 Voir sur le site de la Cinémathèque Française une liste des ouvrages d’Eisenstein et sur Einstein déjà publiés en Russie, en Italie et dans d'autres pays. <http://www.cinematheque.fr/sites-documentaires/eisenstein/rubrique/ressourcesbibliographie.php>.
} 
1920, le grand livre sur la mise-en-scène théâtrale, d'autres textes sur la mise-en-scène, les textes des années 1920 sur le mouvement expressif et le grand volume des " Mémoires ». Donc, les italiens ont à leur disposition cette vaste collection qui respecte beaucoup la manière dont Eisenstein avait conçu ses textes.

C'est à mon avis ça qui explique pourquoi en Italie il y a beaucoup de recherche sur Eisenstein. Notamment Pietro Montani, philosophe qui enseigne l'esthétique à Rome, qui a été celui qui a dirigé cette collection. Et après, à part moi, il y a aussi d'autres chercheurs comme Alessia Cervini, Daniele Dottorini, Francesco Pitassio, Carmelo Marabello et beaucoup d'autres qui ont travaillé sur Eisenstein, grâce à cet accès privilégié que nous avons vu.

La situation est plus compliquée dans d'autres pays, même si maintenant il y a une nouvelle génération de chercheurs qui sont très souvent russes d'origine et qui se sont installés après aux États-Unis ou en France, etc., et qui peuvent donc faire un travail d'archive. Je pense à Ada Ackerman en France, à Elena Vogman en Allemagne, ou à Natalie Ryabchikova aux Etats-Unis.

Est-ce qu'il y a des traductions en cours en français, anglais ou en d'autres langues ? Je pense surtout à « Metod ».

De « Metod », malheureusement, il n'y a pas de traduction en cours en France, mais il y a une traduction en cours en Allemagne, menée par un couple de chercheurs, Georg Witte et Elena Vogman, de la Freie Universität Berlin. Ce sera une édition très importante, parce qu'elle sera probablement aussi un peu différente des deux éditions qui sont parues en russe, parce que cela est une autre complication : parfois les russes ont édités plusieurs versions d'un même volume. Par exemple, « Metod » est paru dans les dernières années en deux éditions assez différentes l'une de l'autre, par Naoum KLEJMAN (2002a et 2002b) d'un côté, et Oksana BULGAKOWA (2008) de l'autre.

Donc, tout ça à mon avis explique pourquoi nous sommes face à un auteur dont le corpus on le connait petit à petit, de manière différente dans les différents pays, et, voilà, c'est ça qui explique...

La chose finale qu'on peut ajouter c'est qu'Eisenstein lui-même avait une vraie difficulté à terminer ses projets. Non seulement à cause d'un manque de temps : il était aussi un cinéaste qui travaillait à beaucoup de films extrêmement complexes et qui a produit aussi beaucoup de projets de films qui ne sont pas achevés. Mais il y a aussi, je dirais, un caractère 
intrinsèque à l'écriture d'Eisenstein, qui fait ainsi qu'elle ait une tendance vers la prolifération d'exemples, la multiplication d'exemples, de références, etc. et qui est une certaine difficulté à boucler de manière définitive dans un projet.

Cela me frappe beaucoup... II me semble qu'il y a une différence de style par rapport aux films et aux écrits d'Eisenstein, parce que dans les films il y a un contrôle presque total de tous les éléments, et en plus il met tout ensemble pour faire une totalité organique pour conduire à l'extase. Mais dans les écrits il y a des choses qui semblent avoir été pensées au cours de l'écriture même. Eisenstein développe un sujet, après il retourne à l'antérieur, etc. Bien, êtesvous d'accord qu'il y a cet écart entre le style des deux ? Le style des écrits peut-il être considéré comme une espèce de réalisation possible du projet du livre sphérique qu'il avait pensé à l'occasion de l'expo Film un Foto, de 1929, en Allemagne ?

Oui, c'est une remarque très juste que vous faites, parce que dans ses films en général Eisenstein a laissé peu ou pas du tout de place à l'aléatoire, à la chance. Il y a vraiment une esthétique du contrôle complet et total de tous les éléments de la représentation. Par rapport à Vertov, qui était vraiment ouvert à cette idée d'un cinéma capable de montrer la vie telle qu'elle se déroule face à l'objectif, une vie qui bien sûr après était remontée de manière très complexe par Vertov, mais que, quand même, chaque plan contenait des éléments de l'aléatoire. Chez Eisenstein il y a une tendance au contrôle total et ce contrôle total trouve son point d'arrivée emblématique dans un film comme «Ivan, le Terrible », qui se déroule presque entièrement dans des espaces clos, fermés, où chaque élément de l'espace est clairement organisé, même en termes de fresque, de dessins, de compositions des plans, etc. par Eisenstein.

Donc, si d'un côté dans ses films il a presque toujours poursuivi cette esthétique du contrôle total, la seule exception étant "Que viva Mexico!», bien sûr, parce que là Eisenstein a agi presque comme un ethnologue qui utilise le cinéma comme un instrument pour mieux comprendre cette société qui le fascinait tellement. Si donc dans le cinéma il met en place cette esthétique du contrôle total, dans les textes écrits, surtout dans les textes qui n'étaient pas prévus pour des publications officielles, mais qui étaient conçus plutôt comme réflexion personnelle en vue d'un livre pas encore achevé...

\section{Par exemple?}

Presque tous les textes de « Metod » l'ont été... Je vais revenir là-dessus, parce qu'il y a une distinction importante. Donc, dans beaucoup de ses textes Eisenstein utilise plutôt 
une sorte de méthode basée sur un flux continu de pensée, un enchaînement d'associations libres, de passages libres, et il y a un peu le modèle, si on veut, de l'écriture automatique surréaliste. C'est- à-dire, laisser que ce soit le flux des associations, des exemples qui surgissent à l'esprit qui constituent le fil rouge de l'argumentation. Donc, voilà, esthétique du contrôle total dans les films, et esthétique du flux d'écriture, du flux de pensé, et parfois, dans certains cas, de l'écriture automatique dans les écrits.

Or, quand on parle des écrits, il faut distinguer nettement à mon avis les textes prévus pour des publications, disons, publiques, officielles, et les textes conçus plutôt comme pages dans lesquelles Eisenstein élabore des idées en vue des livres qui seront peutêtre publiés au futur, mais qui n'étaient pas nécessairement publiables dans le contexte de l'Union Soviétique de l'époque. Il faut se rappeler qu'Eisenstein ne se trouvait pas dans un contexte caractérisé par la liberté totale d'expression. Tout ce qui était public en Union Soviétique surtout aux années 1930 et 1940 était public dans un espace d'une certaine manière dangereux, parce qu'on connaît bien la situation pendant le régime stalinien, les risques auxquels s'exposaient les artistes, les écrivains, les cinéastes, les metteurs en scène qui choisissaient des voies qui ne s'alignaient pas à certaines idées directives de la part du régime.

Et donc il y a plusieurs textes d'Eisenstein dans lesquels Eisenstein lui-même écrit en petit : "for foreign publication », ce qui veut dire "pour une publication à l'étranger ». Il savait que, par exemple, parler, faire l'éloge de la régression psychique, biologique, sociale etc. était quelque chose d'idéologiquement impossible, intenable dans l'Union Soviétique des années 1930 et 1940. Et donc presque tous les textes rédigés pour le livre « Metod» étaient des textes impubliables.

Donc, cette différenciation entre une esthétique du contrôle total et de l'achèvement et une esthétique de l'ouverture, du flux etc., est aussi quelque chose qui distingue les œuvres, films ou textes, prévus pour l'espace public et ceux qui restaient dans l’espace privé ou qui étaient destinés à une publication future ou dans d'autres contextes. 
Dans " La non-indifférente nature ", quand Eisenstein parle sur l'extase cinématographique, il est très clair quand il dit que l'extase est obtenu à partir de l'organique. Donc, il faut avoir une organisation pour qu'on puisse achever l'extase, le niveau maximum du pathos. Cela me parait très original et différent par rapport à d'autres approches. Chez Eisenstein il n'y a pas d'opposition, parce qu'il faut tout organiser, y compris à travers la narrative, pour achever le maximum corporel, c'est-à-dire, l'extase.

Donc, le livre «La non-indifférente nature » est effectivement centré en trois concepts: organicité, pathos, extase. Entre ces trois concepts il n'y a pas du tout d'opposition, de polarité, mais plutôt une certaine cohérence. Pour quoi ça ? Parce que la notion d'organicité, ou d'organique, élaborée par Eisenstein n'est pas une notion qui voit dans l'organisme ou dans l'organisation quelque chose de stable, de statique, de rigide, de fixe. Parfois, quand on parle d'organicité on parle d'harmonie d'éléments qui ont un certain équilibre, une certaine symétrie. On note par exemple que les organismes vivants sont très souvent caractérisés par une certaine symétrie, un certain équilibre, etc. Cette idée d'un organisme comme forme équilibrée et symétrique a été très souvent présente dans l'histoire de l'esthétique, dans l'histoire de la théorie de l'art.

Or, Eisenstein fait référence à une vision différente de l'organisme et de la nature qui à mon avis vient d'Engels et de son livre sur la « Dialectique de la nature ». C'est un livre traduit en 1925, de l'allemand en russe, et qui influencera beaucoup Eisenstein, parce que dans ce livre on trouve l'idée d'une nature comme un ensemble dynamique, toujours instable, toujours en devenir, de processus dialectique, de tensions dialectiques entre des thèses, des antithèses, qui produisent des synthèses toujours précaires, qui continuent, etc.

Donc la vision qu'Eisenstein a de la nature, dans « La non-indifférente nature » est justement d'une nature qui n'est pas indifférente, d'une nature qui est active, qui est traversée par des processus dynamiques, dialectiques. Et par des processus caractérisés par la présence d'une énergie, qui est toujours relâché par les transformations naturelles. Donc, une telle notion d'organicité fait ainsi qu'elle puisse être reliée de manière assez directe avec l'idée de pathos et d'extase, parce que, finalement, le pathos, c'est quoi ? Le pathos indique bien sûr la charge émotionnelle, émotive de quelque chose. La forme organique pour Eisenstein est une forme traversée par plusieurs énergies et capable d'activer les émotions, les passions du spectateur. Donc, la forme organique est pathétique.

Ces deux idées se relient au concept d'extase, parce qu'Eisenstein interprète la notion d'extase en revenant à son étymologie en grec, au fait que le mot extase vient du grec, de deux mots grecs, qui sont $e k$ et stasis. Ek est une préposition qui en grec ancien veut dire 
" au dehors de » et stasis est un substantive qui indique la " stase », une condition de l'extaticité, de l'immobilité. Donc, la forme extatique est une forme caractérisée par une tendance à sortir au dehors de soi, au dehors d'un état d'extase. Donc, organique, pathos et extase sont trois notions étroitement liées et qui indiquent l'idée d'une forme artistique instable, capable de présenter des transformations continues et de relâcher l'énergie qui se transmet ensuite au spectateur.

Ces sont, encore une fois, des concepts à travers lesquels Eisenstein pense le problème qui l'a toujours occupé, celui de comment agir sur les spectateurs à travers la forme.

Eisenstein a parlé à propos de plusieurs possibilités d'arriver à l'extase, soit l'alcool, les corridas, la religion ou le cinéma, par exemple. Mais il voulait toujours que le retour en arrière produit à travers l'extase cinématographique soit productif. Je crois que c'est le sens de votre proposition, comme vous dites dans votre livre, " la dimensione utopica del regresso ". Donc, si on prend "La ligne générale ", par exemple, il y a cette séquence déjà célèbre qui met en parallèle l'extase de la religion et l'extase proprement révolutionnaire. Donc, l'extase peut être aussi dangereux...

Oui, oui, bien sûr. Eisenstein n’a présenté qu’un des textes écrits pour « Metod», celui qui s'appelle, en anglais, « Film form : new problems » (EISENSTEIN, 1949), traduit en français comme «La forme cinématographique: nouveaux problèmes» (EISENSTEIN, 1976). Dans cette occasion, c'était début 1935, Eisenstein se rend compte du fait que son idée de la régression, son idée de l'extase, n'étaient pas du tout acceptables dans le contexte politique et idéologique de ces années-là. Dans ses écrits successifs, il réfléchit sur comment distinguer une dimension productive de l'extase d'une dimension régressive au sens négative du terme. Donc, une dimension de l'extase qui mène vers un irrationnel qui ne conduit nulle part, qui n'est pas utilisée avec un but. Eisenstein s'est toujours intéressé à l'irrationnel, au prélogique, au pathétique, à l'extatique, mais toujours avec une visée d'une certaine manière constructive, c'est-à-dire, avec l'idée que tous ces phénomènes d'irrationalité, d'ilogicité etc., devraient être utilisés dans le champ de l'art pour produire quelque effet clairement conçu, clairement voulu par l'artiste.

Quand vous dites que je parle dans mon livre de la dimension utopique de la régression, là je parle d'une autre chose et je souligne le fait qu'Eisenstein dans ses écrits pour « Metod » s'est intéressé souvent à des formes de régression qui mènent vers des états d'indifférenciation. Par exemple, la régression vers la pensée prélogique est une régression 
vers une forme de pensée qui ne distingue pas nettement, par exemple, entre ce qui est animé et ce qui est inanimé. Il s'agit d'une forme de pensée qui ne distingue pas vraiment entre des individus séparés, mais qui conçoit les individus comme partie d'une sorte de, voilà, esprit commun, communauté, etc. Ou, encore toujours dans les écrits de « Metod », il s'intéresse à des états de régression qui mènent vers des formes biologiques ou des formes de vie encore une fois basées sur l'indifférenciation.

Pourquoi ces formes de régressions seraient-elles utopiques? Parce que, en renvoyant vers ces états d'indifférenciation, en arrière, elle renvoie aussi, en même temps, en avant, vers ce qui était le but politique du socialisme. Ça veut dire, une société conçue sur la base d'un dépassement complet de la différence entre les classes.

Et donc, finalement, Eisenstein semble s'intéresser à la régression en arrière comme une manière d'opérer, d'une manière un peu paradoxale, une projection vers le futur, vers l'idéal du communisme.

Il fallait surtout séparer les différentes formes d'extase. Cela me frappe beaucoup quand il dit, dans "La non-indifférente nature ", qu'il y aurait ces grands artistes qui pourraient voir uniquement un pathétique tragique, comme Victor Hugo, Michel-Ange, etc., parce que les conditions matérielles de l'époque ne leur permettraient pas de voir l'extase et le pathétique comme dans d'autres époques ${ }^{5}$. Je crois que c'est cela qui s'est passé avec le cinéma brésilien du début des années 2000. C'est une extase, il y a une telle puissance, mais c'est tragique ${ }^{6}$.

Oui, oui, parce que finalement Eisenstein va chercher dans l'histoire de l'art, dans l'histoire des formes, des religions, des exemples d'extase qui puissent être éventuellement réactivées au présent, ou prises comme point de référence, mais il était très conscient du fait que chacun de ces exemples d'extase était situé dans un contexte politique, idéologique précis. C'est seulement, à son avis, dans le contexte idéologique de l'Union Soviétique et du socialisme qu'on peut trouver un élément progressif dans cette idée de régression vers l'indifférencié.

Après il est légitime de se poser la question si Eisenstein croyait vraiment à ce qu'il écrivait dans ses textes, parce que, comme je disais, la question de la dimension politiques de ses textes doit être toujours abordée par rapport au contexte particulier dans lequel il écrivait. C'était un contexte dans lequel Eisenstein se posait toujours le problème de

\footnotetext{
5 Voir « La non-indifférente nature 2 », notamment les pages 288-312 (EINSENSTEIN, 1978).

6 Il s'agit d'une thèse en développement par l'interviewer qui se rapporte, entre autres, aux films de Beto Brant, Sergio Bianchi et Claudio Assis sortis au début des années 2000 au Brésil.
} 
comment rendre ses idées, ses théories, acceptables dans l'espace public. Comment créer des ponts, des médiations, des liaisons, entre ses propres idées et le discours officiel.

Par contre, si on prend "Que viva Mexico ! ", par exemple, je ne crois pas qu'il était soumis.

Non, c'est le seul cas dans lequel il travaille un projet presque totalement indépendant. C'est vrai qu'il avait reçu un financement d'Upton Sinclair, qui avait aussi envoyé le frère de son épouse à suivre la production du film, donc Eisenstein avait un peu ce regard du contrôle, mais il ne prêtait pas beaucoup d'attention à ça... Donc, «Que viva Mexico! » est un projet assez indépendant. Mais dans tous les autres cas, pendant qu'il est en Union Soviétique, Eisenstein s'est toujours posé le problème de comment créer des médiations entre son propre parcours intellectuel et l'espace idéologique officiel.

Avec cela je ne veux pas du tout dire qu'à chaque moment où Eisenstein fait référence au socialisme il est en train de mentir, d'être hypocrite, de dire quelque chose à laquelle il ne croit pas. Non, pas du tout. Je soulève seulement la question qu'il ne s'agit pas de quelqu'un qui écrit dans un contexte d'indépendance presque totale de pensée, mais d'un contexte où la liberté d'expression devait toujours se situer par rapport à d'autres éléments.

Sur la plasmaticité, que chez Eisenstein est une forme importante de retour en arrière. Quand il écrit sur Walt Disney, c'est passionnant ${ }^{7}$. La question que je pose c'est sur "Ivan, le terrible ", les formes de plasmaticité dans ce film. Est-ce que " Ivan, le terrible " est le film le plus plasmatique d'Eisenstein ? Où peut-on voir cette plasmaticité ? Dans la lumière, les ombres, les volumes des corps?

Oui, oui. Eisenstein écrit son texte sur Disney en 1940, dix ans après avoir rencontré effectivement Disney en Californie. Donc, 1940 est avant le début du tournage d'«Ivan, le terrible ", même s'il y a des parties de ce texte qui sont écrites à Almata, où Eisenstein était avec toutes les personnages engagées dans la production d'« Ivan, le terrible ». Donc, tout cela pour dire que ce texte, cette réflexion, a surement influencé le tournage et la conception d'« Ivan, le terrible ». Comment ? Je dirais avec, par exemple, tout le travail sur les ombres, qui est très important, mais aussi avec le travail sur la figure et le visage d'Ivan, le terrible, lui-même. C'est un visage qui évolue beaucoup au cours du film et qui présente des éléments plastiques qui sont

7 "Walt Disney" (EISENSTEIN, 2013). 
un peu invraisemblables, comme cette barbe à pointe, qui est vraiment utilisée de manière presque sculpturale par Eisenstein.

C'est ce qui a gêné Staline.

Voilà, ce qui a gêné Staline. Et il y a aussi une dimension très grotesque dans certains personnages, dans certains des boyards, et aussi dans certains costumes. Il y a la présence d'une dimension liée d'un côté à certaines formes de théâtre, auxquelles Eisenstein s'était intéressé, mais aussi à la bande dessiné etc. Donc, tous ces éléments sont en jeu dans ce film.

Et aussi, comme je disais, le fait que ce film soit tourné presque complètement dans des espaces fermés donne à Eisenstein la possibilité de travailler sur la plasticité de cet espace de manière totale, sans devoir se rapporter à des éléments déjà existants.

Pour moi, c'est important aussi parce que je crois qu'il y a une certaine plasmaticité dans les films que j'étudie, mais ce n'est pas une plasmaticité heureuse et légère, c'est une plasmaticité du sang, de l'urine, de la fumée...

Oui, mais plusieurs auteurs, nous avons organisé il y a quelques années un séminaire sur certaines lectures, interprétations de Disney de la part de figures comme Eisenstein, comme Walter Benjamin et d'autres, et ces textes ont été publiés en russe (SOMAINI, 2013). Et ce qu'on soulignait c'était que beaucoup d'auteurs de ces années-là soulignaient la dimension pas seulement joyeuse, infantile, etc., des bandes dessinées de Disney, mais aussi la dimension monstrueuse, inquiétante. Il y a beaucoup de violence dans le premier Disney, beaucoup de corps soumis à des transformations forcées, beaucoup d'objets qui s'animent et deviennent menaçants. C'est vraiment de la tradition des fables pour enfants, les Frères Grimm, Andersen, c'est une tradition qui a toujours élaboré des contes pour les enfants, mais qui étaient aussi traversés par l'horreur, la terreur, etc.

Donc, ce n'est pas que Disney est seulement un monde heureux. Il y a déjà des éléments chez Disney qui peuvent aller dans des directions comme celle qui sera reprise par Eisenstein dans « Ivan, le terrible». 
Encore sur Disney, on peut voir qu'Eisenstein aime l'expression anglaise, animated cartoon, parce qu'il y a cette chose de l'animisme. Le retour en arrière chez lui est pareil à vivre une expérience pleine de possibilités. II parle de "revivre la toute-puissance-originelle ", "la force élémentaire du devenir ", "la plasmaticité de l'existence, de quoi tout peut surgir ${ }^{8}$.

Je voudrais savoir à propos d'une idée contemporaine d'Eisenstein, sur laquelle je suis en train de travailler, la pulsion de mort chez Freud. Quand Freud découvre cette pulsion de mort, c'est une espèce d'inanimisme, c'est cela qu'il dit, que la pulsion de mort est une force de la matière inanimée qui pousse les êtres vivants vers la matière sans vie, inanimée. Et donc, c'est une sorte de retour en arrière sans la force de l'animisme, mais peut-être avec la force d'un inanimisme, disons, d'une pulsion de mort ${ }^{9}$.

Oui, Eisenstein s'est beaucoup intéressé aux théories de certains élèves de Freud, comme Otto Rank et Sándor Ferenczi. Ces sont des psychanalystes élèves de Freud, mais qui dans un certain moment seront désavoués par Freud lui-même, qui les considérera un peu comme... hérétiques, pour avoir pris des voies qui s'éloignaient de sa pensée. Ces deux auteurs ont traité, de manière un peu différente, une même idée qui est en liaison avec le todestrieb, la pulsion de mort chez Freud. C'est-à-dire le désir de retour dans le ventre maternel, dans le sein maternel. Et ce retour est conçu par les deux comme une volonté de retour à un état originaire, duquel on a été forcés à travers une rupture traumatique, qui est la rupture de la naissance, le traumatisme de la naissance, comme a dit Otto Rank. Et parfois ce désir de retour en arrière est conçu aussi par ces auteurs comme un désir de mort, parce que finalement le retour dans le ventre de la mère est une sorte d'annulation de soi et un retour à une dimension préconsciente qui a des analogies avec la mort, finalement : une forme d'annulation, de perte de soi.

Eisenstein s'est intéressé à ces auteurs, il a traité beaucoup de leurs théories dans ses écrits et il a utilisé cette idée de manière très explicite dans la deuxième partie d'« Ivan, le terrible ». À la fin de la scène de la fête des opritchniki, la séquence en couleur, le jeune Vladimir, auquel on a mis une couronne, comme une sorte de couronnement fictif, carnavalesque, est invité à se diriger vers la cathédrale. Et on voit Vladimir, qui s'approche à la porte qui fait sortir de l'espace de la fête vers l'espace de la cathédrale et cette porte est conçue par Eisenstein vraiment comme une porte, un seuil qui reconduit à l'intérieur du ventre de la mère. C'est le retour en arrière, mais c'est aussi une allée vers la mort. Si vous regardez cette scène-là, Eisenstein souligne beaucoup l'état d'horreur, de froid, éprouvé par

\footnotetext{
8 "Walt Disney" (EISENSTEIN, 2013, p. 64).

9 «Si nous admettons, comme un fait expérimental ne souffrant aucune exception, que tout ce qui vit retourne à l'état inorganique, meurt pour des raisons internes, nous pouvons dire : la fin vers laquelle tend toute vie est la mort ; et inversement le non-vivant est antérieur au vivant. » FREUD (2002, p. 35).
} 
ce personnage face à ce seuil. Et l'espace de la cathédral dans la scène suivante fut explicitement conçu par Eisenstein comme un espace qui devrait évoquer l'utérus, qui devrait vraiment évoquer le retour à l'intérieur du ventre de la mère.

Et voilà, si vous prenez le petit livre d'Yuri Tsivian (2001), en anglais, «Ivan, the terrible », édité par le BFI, British Film Institut, c'est un petit livre qui est magnifique. Dans ce livre-là, il parle de cette scène que j'ai évoquée.

La question de la pulsion de mort comme retour vers l'inorganique, vers l'inanimé etc., n'est pas présente, à mon avis, chez Eisenstein. On trouve ça beaucoup plus chez Kracauer. Son texte "Theory of film» (KRACAUER, 1997) et surtout les matériaux préparatoires à ce texte (KRACAUER, 2005), peuvent être mis en relation avec le Todestrieb de Freud, tandis que chez Eisenstein ces deux auteurs-là, Rank et Ferenczi, sont beaucoup plus présents.

Dans votre livre, vous donnez beaucoup d'importance aux dessins. Je vous questionne si les dessins ont valeur comme des œuvres autonomes ou s'ils sont surtout importants pour comprendre la personnalité d'Eisenstein, pour comprendre les films et les écrits. Et, aussi, pour mélanger un peu la question, les dessins sont beaucoup plus agressifs, ou bien sont agressifs d'une autre façon. Si on prend "Que viva Mexico ! ", par exemple, la mort est plus allégorique, tandis que dans les dessins, la mort est viscérale.

Alors, à mon avis, les dessins, et quand on dit "dessins d'Eisenstein », on parle de centaines et centaines de dessins qui se trouvent dans plusieurs archives et parfois dans des collections qui viennent à connaissance de temps en temps. Donc, c'est une collection encore plus énorme qui, à mon avis, doit être considérée à la même hauteur d'importance des films et des textes. Ça fait vraiment partie de l'œuvre d'Eisenstein à part entière.

Et ce qui est intéressant avec les dessins, d'un côté est leur évolution dans le temps. Eisenstein commence à dessiner beaucoup déjà quand il était enfant, après il dessine beaucoup dans les années dans lesquels il est metteur en scène de théâtre au début des années 1920, après il arrête de dessiner pour quatre ou cinq ans, quand il fait ces films comme "La grève ", « Le cuirassé Potemkine » etc. Et finalement il reprend au Mexique, où il dessine énormément, et il continue jusqu'à la fin de sa vie.

Donc l'intérêt de ses dessins est pluriel. D'un côté, il s'agit des dessins qui nous aident à comprendre comment Eisenstein concevait ses projets, de mise-en-scène, de réalisation, etc. 
Mais de l'autre, ce sont des dessins très souvent privés, tout à fait privés, qu'il donnait parfois à des amis comme cadeau.

\section{II ne voulait pas les publier?}

Il n'avait pas vraiment pensé les publier, sauf une fois au Mexique, sous invitation d'une jeune américaine, qui s'appelait Anita Brenner, qui pensait organiser une exposition, mais là c'était un cas particulier, parce que ça aurait été une exposition aux États-Unis et dans une condition de liberté d'expression plus grande que celle d'Union Soviétique.

Et voilà une situation dans laquelle des dessins avec des formes et des éléments disons, surréalistes, comme ceux d'Eisenstein au Mexique, auraient pu être présentés, tandis qu'en Union Soviétique, ça aurait été complètement inacceptable.

Donc, l'intérêt des dessins est de voir sa pensée en action dans un espace privé et avec une liberté qu'il n'avait pas quand il s'agissait des textes et des films pensés pour un espace public.

Le dessin est une activité privée, est une activité rapide, de réflexion très rapide, encore une fois basée sur le modèle du flux inconscient, de l'écriture automatique, etc. Et donc il y a aussi des dessins qui ont une valeur purement théorique. Ce sont des dessins qui ne sont pas liés à des projets concrets de mises-en-scène ou de films, ils sont élaborés pour penser la nature du montage, ou encore pour penser la nature de certains projets théoriques sur lesquels Eisenstein travaillait. Quand je parle des dessins conçus comme des réflexions sur le montage, je fais référence surtout à la série magnifique qui concerne « La mort du Roi Duncan ». C'est une série qui commence en 1930 et qui continue pendant l'été 1931, quand Eisenstein était en train de tourner l'épisode Maguey de « Que viva Mexico ! ». Là Eisenstein fait des séries de dessins dans lesquelles il retravaille constamment, de manière toujours différente, la relation entre ces trois personnages, le Roi Duncan, Macbeth et Lady Macbeth.

\section{Est-ce qu'il était en train de produire ou concevoir quelque travail autour de Shakespeare ?}

Non, il avait fait un projet sur Macbeth pour un spectacle théâtral au début des années 1920 et cette scène de meurtre est toujours restée pour lui une scène emblématique, parce que dans sa pensée l'idée de montage est aussi liée á l'idée du meurtre, à l'idée de la coupe, à l'idée de la blessure, la coupe qui sépare et qui réorganise. Ce sont des dessins dans lesquels le corps du Roi Duncan est démembré, soumis à toute sorte de transformation et 
c'est vraiment une réflexion sur la plasticité, une réflexion sur comment organiser la forme à l'intérieur de l'espace rectangulaire de la page. À mon avis, des dessins comme ça sont vraiment un exemple de dessins théoriques, utilisés pour penser le montage.

Croyez-vous que la coupe en morceaux, qu'on voit dans les dessins, c'est aussi un type de plasmaticité ?

Oui, plasmaticité évoque l'idée de, disons, transformer un matériel de base et la transformation peut succéder de plusieurs formes, à travers une sorte de manipulation, ou à travers un travail qui élimine des parties, comme dans la sculpture, ou encore à travers la coupe et le remontage.

En ce qui concerne l'œuvre "La non-indifférente nature ", est-ce qu'elle peut être une réaction à une certaine conception liée au néoréalisme, qui était déjà en vue d'apparaître ? Parce qu'on y trouve la défense passionné de la non-indifférence de la nature.

Je ne sais pas, parce qu'Eisenstein était en train d'écrire ce livre entre 1941 et 1945, donc dans des années qui précèdent le néoréalisme italien. Même s'il mentionne « Rome, ville ouverte » dans son texte sur le cinéma en relief, sur le cinéma stéréoscopique. Donc, en 1946, 1947 il connaissait, il avait vu quelque chose parmi les films néoréalistes italiens, mais je ne dirais pas du tout que l'idée de la non-indifférente nature est conçue comme une réaction à ça. C'est une idée pour lui de poursuivre sa recherche sur la forme efficace à travers de nouveaux concepts comme, voilà, organicité, pathos, extase, et de reprendre tout son intérêt pour le pathos, l'extase, qui était déjà présent dans les années 1920, parce que « La ligne générale », la scène de l'écrémeuse, elle est déjà une scène... et voilà, on voit que dans « la non-indifférente nature » il y a un chapitre dédié à ça. 
Pour finaliser, je voudrais vous écouter un peu sur l'actualité de la pensée d'Eisenstein. Pour moi c'est remarquable que vous mettez Eisenstein toujours en relation avec plusieurs artistes et théoriciens: Benjamin, Kracauer, Bataille, etc. Et dans vos cours à Paris 3, vous faites la relation avec Mcluhan et l'archéologie des médias. Donc, Eisenstein serait parmi les auteurs et artistes de référence pour un abordage contemporain du médium. Croyez-vous que le principal est d'investir et peut-être de récupérer l'œuvre d'Eisenstein ? Et croyez-vous que les derniers concepts d'Eisenstein, tels que l'extase, la plasmaticité et la pensée prélogique, sont encore nécessaires aujourd'hui ?

Il y a un double intérêt qui a parcouru mon travail sur Eisenstein. D'un côté, un intérêt visant mieux situer la figure d'Eisenstein dans les années 1920, 1930 et 1940 et le faire sortir d'une colocation exclusive à l'intérieur de l'histoire et de la théorie du cinéma, pour le situer dans un réseau plus vaste d'autres penseurs de cette période avec lesquels parfois il n'était pas entré en contact. Il n'a jamais rencontré Benjamin, il n'a jamais rencontré Warburg, il n’a jamais rencontré Ernst Bloch, mais on peut trouver des éléments, des affinités, des rencontres, des croisements, extrêmement intéressants. À partir par exemple du fait qu'Eisenstein et Warburg, tous les deux, donnent une importance centrale à l'idée de formule de pathos, dans deux contextes très différents l'un de l'autre, mais quand même ils soulignent la centralité de ces concepts. Donc, d'un côté, et c'est surtout ce que j'ai essayé de faire dans mon livre, j’ai essayé de situer Eisenstein dans ce vaste réseau des années 1920,1930, 1940, en discutant des relations avec d'autres auteurs qui n'ont pas jusqu'ici été abordés.

De l'autre côté, il y a l'intérêt d'Eisenstein par rapport à notre propre situation aujourd'hui dans la théorie des médias, des images et du cinéma. Et alors là il me semble qu'Eisenstein a une actualité remarquable, parce que, par exemple, dans une phase comme la nôtre, dans laquelle nous nous interrogeons beaucoup sur les transformations du dispositif cinématographique, sur le rapport entre le cinéma et l'histoire des arts, des arts plastiques etc., toute la pensée d'Eisenstein, si on veut, a tourné aussi autour de cette question. Il s'est toujours intéressé à comment le cinéma pouvait entrer en relation avec l'histoire du théâtre, de la peinture, de la sculpture, de la littérature, en reformulant sur son propre terrain, à travers l'instrument du montage, des questions qui s'étaient posées dans d'autres domaines artistiques.

Voilà donc une première raison pour l'intérêt d'Eisenstein pour cette question de la relation du cinéma avec les autres arts et de la transformation dans le temps du dispositif cinématographique. Après il y a une autre raison de s'intéresser et cela concerne son approche de l'histoire. Les études cinématographiques ont vu depuis une quinzaine, une 
vingtaine d'années, la parution d'un courant, qu'on a appelé New film history dans les pays anglophones, un courant qui a complètement remis en question certains des principes, certaines des catégories qui régissaient la réflexion historique sur le cinéma. On a souligné, par exemple, comment le cinéma a toujours été parcouru par plusieurs lignées généalogiques qui le mettaient en relation avec plusieurs traductions, le vaudeville, le music-hall, le théâtre etc.

Et ce dernier projet, des «Notes pour une histoire générale du cinéma» (EISENSTEIN, 2014a) ${ }^{10}$, qu'on vient de publier, à mon avis peut être mis en relation de manière très intéressante avec ce courant de la New film history. Donc, Eisenstein pourrait être lu comme un certain précurseur : c'est un mot toujours un peu difficile à utiliser, mais en tout cas, penseur à mettre en relation avec cette nouvelle approche de l'histoire du cinéma.

\section{À travers l'idée d'imagicité ou de cinématisme ?}

Cinématisme, c'est un concept qui n'a pas été utilisé tel quel par Eisenstein. C'est un concept qui a été utilisé pour le titre du recueil publié par Albera (1980/2009). Mais c'est un concept qui indique très bien cette idée d'Eisenstein de retrouver des éléments de cinématographicité dans d'autres arts. Le terme qu'Eisenstein a utilisé en russe est kinematographitchnost, qui veut dire cinématographicité. Ce n'est pas exactement cinématisme, mais c'est essentiellement la même chose : des éléments du cinéma avant le cinéma.

La dernière raison pour laquelle à mon avis Eisenstein est très intéressant est parce qu'on trouve dans sa pensée des éléments d'une anthropologie des images, d'une esthétique anthropologique des images. Ça veut dire, toute une réflexion sur comment les images se relient à certains besoins, certaines impulsions, certaines nécessités de l'homme et comment les images interviennent dans l'ensemble de l'expérience humaine, en produisant des effets, en assurant la possibilité de réserver dans le temps certains phénomènes, d'aider la mémoire, de contribuer à la remémoration du passé, à la possibilité de revivre, de remettre en scène le passé, etc. Donc, aujourd'hui, on revient beaucoup sur cette question de l'anthropologie de l'image, de l'anthropologie du cinéma, on peut mentionner plusieurs ouvrages et plusieurs colloques qui se sont tenus récemment sur ce sujet et là encore

${ }^{10}$ Le livre est aussi paru au Brésil (EISENSTEIN, 2014b). 
Eisenstein a des positions très intéressantes. Donc, à mon avis, c'est un penseur dont l'importance actuelle est tout à fait semblable à celle de Walter Benjamin, de Aby Warburg ou d'autres figures qu'on relit et qu'on étudie toujours beaucoup .

\section{Références}

EISENSTEIN, Sergei. Cinématisme: Peinture et cinéma. Édition établie par François Albera. Bruxelles, Complexe, 1980.

EISENSTEIN, Sergei. Film form: essays in film theory. New York, Harcourt, Brace and Co., 1949.

EISENSTEIN, Sergei. Film form: new problems. In: Film form: essays in film theory. New York: Harcourt, Brace and Co., 1949.

EISENSTEIN, Sergei. La forme cinématographique: nouveaux problèmes. In: EISENSTEIN, Sergei. Le film, sa forme, son sens. Paris, ed. Christian Bourgois, 1976.

EISENSTEIN, Sergei. La méthode - Grundproblem. Sous la direction de Naoum Klejman. Moscow : Ed. du Musée du cinéma, 2002a. Premier tome.

EISENSTEIN, Sergei. La méthode - Les secrets des maîtres. Sous la direction de Naoum Klejman. Moscow: du Musée du cinéma, 2002b. Deuxième tome.

EISENSTEIN, Sergei. La non-indifférente nature 2. Paris: Union générale d'éditions, 1978.

EISENSTEIN, Sergei. Le mouvement de l'art. Édition établie par François Albera. Paris : du Cerf, 1986.

EISENSTEIN, Sergei. Les gendarmes à la Sorbonne. L'humanité, Paris, 18 févr. 1930a.

EISENSTEIN, Sergei. Les principes du nouveau cinéma russe. La revue du cinéma, Paris, $n$. 9, 1 avr. 1930b.

EISENSTEIN, Sergei. Metod / Die Methode. Sous la direction de Oksana Bulgakowa. Berlim: Potemkim, 2008.

EISENSTEIN, Sergei. Notes pour une histoire générale du cinéma. Édition établi par Antonio Somaini et Naum Kleiman. Paris: ed. AFRHC, 2014a.

EISENSTEIN, Sergei. Notas para uma história geral do cinema. Edição estabelecida por Naum Kleiman et Antonio Somaini. Rio de Janeiro: Azougue, 2014b.

EISENSTEIN, Sergei. The film sense. London: Faber and Faber, 1943. 
EISENSTEIN, Sergei. Walt Disney. Belval: Circe, 2013.

FREUD, Sigmund. Au-delà du principe de plaisir. Édition numérique. Chicoutimi : J. M. Tremblay, 2002.

KRACAUER, Siegfried. Theory of film: the redemption of physical reality. Princeton: Princeton University, 1997.

KRACAUER, Siegfried. «Marseiller Entwurf» zu einer Theorie des Films (1940). In: KRACAUER, Siegfried. Theorie des Films. Die Errettung der äußeren Wirklichkeit. Frankfurt: Suhrkamp, 2005.

SOMAINI, Antonio. Ejzenštejn. Il cinema, le arti, il montaggio. Torino: Piccola Biblioteca Einaudi, 2011.

SOMAINI, Antonio. Généalogie, morphologie, anthopologie des images, archéologie des médias. In : EISENSTEIN, Sergei. Notes pour une histoire générale du cinéma. Édition établi par Antonio Somaini et Naum Kleiman. Paris: AFRHC, 2014.

SOMAINI, Antonio. Kollektivnyj smech kak “terapevtičeskij vyplesk bessoznatel'nych energij”. Ben'jamin o Disnee. Kinovedcheskie zapiski, n. 104-15, p.194-202, 2013.

TSIVIAN, Yuri. Ivan, the terrible. London : British Film Institute, 2001. 


\title{
Eisenstein contemporâneo: entrevista com Antonio Somaini
}

\section{Resumo}

Entrevista com Antonio Somaini sobre os filmes, os textos e os desenhos de Sergei Eisenstein. Somaini é atualmente um dos grandes especialistas em Eisenstein. Nesta entrevista, conversamos sobre variados assuntos: o desafio de traduzir as obras de Eisenstein; as fases da carreira; o desenvolvimento do pensamento; a relação dos filmes com os textos; a paixão pelos desenhos de Walt Disney; e a prática do desenho pelo próprio Eisenstein. Trata-se de uma longa entrevista onde falamos também de alguns dos principais conceitos de Eisenstein, tais como êxtase, pathos, organicidade, atração, regressão e plasmaticidade. Somaini esclarece, ainda, a especificidade do seu trabalho e a urgência de voltarmos a Eisenstein. De um lado, é preciso retirá-lo da posição exclusiva que ocupa na história do cinema e inseri-lo na rede mais vasta das artes e do pensamento dos anos 1920, 1930 e 1940. De outro, é preciso pensar seu projeto como essencialmente contemporâneo. Como nos diz Somaini, isto que pensamos bastante atualmente, o dispositivo cinematográfico, a relação entre o cinema e a história da arte, a influência das imagens na experiência humana, todas essas questões sempre foram o problema central em Eisenstein.

\section{Palavras-chave}

Eisenstein. Somaini. Extase. Pathos. Plasmaticidade.

\section{Contemporaneous Eisenstein: interview with Antonio Somani}

\begin{abstract}
Interview with Antonio Somani about the movies, texts and the drawings of Sergei Eisenstein. Somani is nowadays one of the greatest specialists in Eisenstein. In this interview we talked about a variety of subjects: the challenge of translating the works of Eisenstein; the career stages; the development of thoughts; the relation between the movies and texts; the passion for Walt Disney drawings; and about the drawing practice of Eisenstein himself. It is a long interview where we also talked about some of Eisenstein main concepts, such as ecstasy, pathos, organicity, attraction, regression and plasticity. Somani also clarifies about the specificity of his work and the urgency of going back to Eisenstein. On one side, we need to remove him from the exclusive position that he occupies in the history of cinema and insert him in a wider network of the arts and thoughts of the twenties, thirties and forties. On the other side, we need to think of his project as essentially
\end{abstract}


contemporary. As said by Somani, what we think currently, the cinematic apparatus, the relationship between cinema and the history of art, the influence of images on human experience, all these questions always were the central problem for Eisenstein.

\section{Keywords}

Eisenstein. Somani. Ecstasy. Pathos. Plasmaticité.

Recebido em 12/04/2016

Aceito em 12/04/2016 